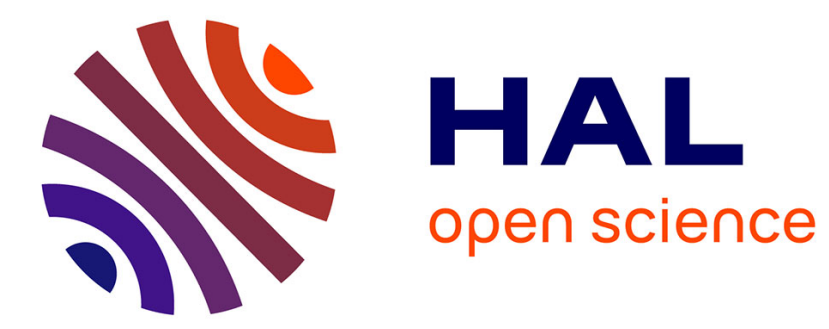

\title{
INFLUENCE DE LA GRANULOMETRIE SUR LA TENACITE DYNAMIQUE D'UN PROPERGOL
}

\author{
R. Neviere, M. Nait-Abdelaziz, G. Pluvinage
}

\section{To cite this version:}

R. Neviere, M. Nait-Abdelaziz, G. Pluvinage. INFLUENCE DE LA GRANULOMETRIE SUR LA TENACITE DYNAMIQUE D'UN PROPERGOL. Journal de Physique Colloques, 1988, 49 (C3), pp.C3-261-C3-268. 10.1051/jphyscol:1988337 . jpa-00227760

\section{HAL Id: jpa-00227760 https://hal.science/jpa-00227760}

Submitted on 1 Jan 1988

HAL is a multi-disciplinary open access archive for the deposit and dissemination of scientific research documents, whether they are published or not. The documents may come from teaching and research institutions in France or abroad, or from public or private research centers.
L'archive ouverte pluridisciplinaire HAL, est destinée au dépôt et à la diffusion de documents scientifiques de niveau recherche, publiés ou non, émanant des établissements d'enseignement et de recherche français ou étrangers, des laboratoires publics ou privés. 
INFLUENCE DE LA GRANULOMETRIE SUR LA TENACITE DYNAMIQUE D'UN PROPERGOL

\author{
R.G. NEVIERE, M. NAIT-ABDELAZIZ et G. PLUVINAGE \\ Laboratoire de Fiabilité Mécanique, Faculté des Sciences, \\ F-57045 Metz Cedex, France
}

Résumé : Le problème de l'amorçage d'une fissure sous sollicitation dynamique est analysé dans le cadre d'une élude expérimentale concernant les carburants solides d'engins spatiaux auto-propulsés. La tenacité est obtenue par l'application de la théorie généralisée de mécanique de la rupture introduite par E.H ANDREWS.

Les résultats montrent une forte sensibilité de la tenacité à la vitesse de déformation et permettent d'obtenir une première approche du processus de rupture particulier à ce matériau.

Abstract : Fracture toughness of a solid propellant is investigated under dynamic loading conditions using a split Hopkinson tensile device.

Since this material exibits non-linear visco-elastic behaviour fracture toughness is estimated using generalized fracture theory proposed by E.H. ANDREWS. Experimental results confirm strong dependence with respect to the combustive particles size distribution as it could be expected when considering high heterogeneity of the material structure.

\title{
1 INTRODUCTION
}

L'application des principes de la thermodynamique a permis, grâce à la très grande généralité des phénomènes qu'ils décrivent, de faire progresser de nombreux domaines de la recherche scientifique. La mécanique de la rupture n'y fait pas exception puisque l'on trouve le premier principe à l'origine du raisonnement de GRIFFITH dès 1920 . Plus récemment c'est aussi un bilan énergétique qui a conduit RICE à formuler un des premiers critères de rupture élasto-plastique qui connait aujourd'hui le succès que l'on sait.

Si, finalement, toutes ces théories ont été introduites en vue de leur application à la rupture des métaux, leur généralisation doit cependant permettre leur utilisation pour tous les matériaux et notament les materiaux nouveaux. On trouve d'ailieurs dans les travaux de RICE de nombreuses références à ceux de RIVLIN et THOMAS [1] sur le caoutchouc naturel. Ces mêmes travaux sont également cités par ANDREWS [2] en illustration de sa théorie généralisée de mécanique de la rupture proposée en 1974. Moins connue que l'intégrale de RICE, cette théorie conduit également à un critère de rupture de type énergétique dont le présent papier se propose de mettre en valeur tout l'intérêt par le biais d'une étude expérimentale réalisée sur les carburants solides utilisés dans les moteurs de lanceurs de satellites et de nombreux engins balistiques auto-propulsés : les propergols.

\section{GENERALITES SUR LE COMPORTEMENT MECANIQUE DES PROPERGOLS}

Ce matériau est un agglomérat de charges cristallines combustives (en général du perchlorate d'amonium) insérées par malaxage à chaud et en fort pourcentage pondéral ( $80 \%$ ) dans une matrice thermoplastique (polybutadienne) tenant lieu de réducteur lors de la combustion. Le problème de la rupture de ce type de matériau se pose dés lors que l'on observe une modification du comportement balistique des engins consécutive à une combustion préférentielle le long de fissures, cette combustion indésirable conduisant parfois même à leur explosion en vol.L'apparition de ces fissures a plusieurs origines mais elles sont le plus généralement dûes aux fortes accélérations du décollage et aux vibrations intenses durant le fonctionnement. 
La caractérisation en termes de mécanique de la rupture de l'amorçage de ces défauts se doit de faire appel aux critères de type énergétique puisque l'on observe un comportement non-linéaire dû à la présence du polymère qui introduit une composante visco-éliastique.

Pour conclure sur le comportement mécanique global de ce matériau, il faut ajouter que l'on observe à partir d'un certain taux de déformation, un phénomène de décohésion liant-charges s'accompagnant d'une variation de volume. L'ensemble de ces caractéristiques et plus particulièrement l'effet de visco-élasticité nous a conduit à ne traiter que le problème de l'amorçage des défauts dans un premier temps.

\section{ETUDE EXPERIMENTALE}

\subsection{Qbjes de l'étude}

La fractographie de la figure 1 met en évidence l'importante hétérogénéité de la structure du matériau tant au niveau de la composition que de la dimension des charges combustives. Ces dernières étant à l'origine de la rupture il était, d'évidence, d'un grand intérêt de faire varier leur répartition granulométrique. De plus, étant donné d'une part le type de sollicitations rencontrées à l'utilisation et d'autre part les effets de visco-élasticité qui rendent sensible ce matériau à la vitesse de déformation, létude expérimentale a été réalisée sous sollicitation dynamique.

\subsection{Matériau testé-échantillens}

Le matériau testé est un propergol inerte (les charges combustibles ont été remplacées par des charges chimiquement neutres de caractéristiques mécanique équivalentes) à liant polybutadienne. Quatre types de répartions granulométriques ont été réalisées, leur composition respective est rappelée dans le tableau ci-dessous. (Remarquer la dimension importante des plus gros grains)

\begin{tabular}{|c|c|c|c|c|}
\hline \multicolumn{2}{|c|}{$\begin{array}{l}\text { Dimension des charges } \\
\text { (\% pondéral) }\end{array}$} & $400 \mu$ & $30 \mu$ & $8 \mu$ \\
\hline Granulomètrie & GO & $50 \%$ & $25 \%$ & $25 \%$ \\
\hline$"$ & G1 & $35 \%$ & $35 \%$ & $30 \%$ \\
\hline$"$ & G2 & $75 \%$ & $12,5 \%$ & $12,5 \%$ \\
\hline Granulométrie & $E$ & $75 \%$ & $0 \%$ & $25 \%$ \\
\hline
\end{tabular}

Tableau A

La géométrie des échantillons est imposée par le dispositif expérimental de traction dynamique (cf. paragraphe 3.3). Il s'agit d'une géométrie axisymétrique de cliamètre $16 \mathrm{~mm}$, hauteur $7 \mathrm{~mm}$ dans laquelle on réalise mécaniquement des fissures annulaires de profondeurs différentes.

\subsection{Dispositif expérimental de traction dynamique}

Ce dispositif a été mis au point spécialement pour l'étude de ce type de matériaux. Il s'agit d'un appareillage inspiré du dispositif de barres d'Hopkinson qui esı aujourd'hui assez répandu pour la réalisation d'essais de compression dynamique [3].

Généralement ce type de dispositif ne s'adapte que difficilement aux essais de rupture puisqu'il est alors nécessaire de transmettre une onde de traction à l'échantillon ce qui pose inévitablement le problème de sa fixation sur les barres sachant que les liaisons par vissage ne transmettent que médiocrement les ondes de contraintes. Citons toutefois les solutions de KLEPACZKO [ 4 ] et de KOLSKY [5] qui permettent de résoudre ce problème de façon élégante. 
Dans notre cas les caractéristiques mécaniques particulières du matériau à teșter permettent de contourner ce problème puisque les faibles charges à rupture (de $10^{2}$ à $\left.10^{3} \mathrm{~N}\right)$ permettent d'envisager une solution par collage. Les échantillons sont donc collés sur des supports du même matériau que les barres a l'aide d'une colle époxy (polymérisation $48 \mathrm{~h}$ ) ce montage étant ensuite lui-même collé entre les barres à l'aide d'une colle cyanoacrilate dont la durée de polymérisation de 5 minutes réduit considérablement le temps de manipulation.

L'ensemble du dispositif ainsi que la chaîne de mesure associée est représentée figure 3. Il est constitué de deux barres en alliage d'aluminium dont le faible module d'Young permet d'augmenter l'amplitude des déformations au niveau des capteurs incident et transmis. Pour la même raison ces capteurs sont constitués de jauges de déformation à semi-conducteur (facteur d'amplification = 100 à comparer avec environ 2 pour les jauges de type résistif).

L'onde de traction est obtenue par l'impact d'un projectile tubulaire sur un talon de faible longueur vissé sur l'extrémité de la barre incidente.

La chaîne de mesure est composée de deux ponts d'extensométrie dynamiques qui transmettent le signal amplifié à la mémoire d'un oscilloscope numérique elle-même transférée dans un micro-calculateur qui reconstitut la courbe charge déplacement puis l'enregistre sur disquette.

Rappelons qu'en négligeant l'inertie radiale, l'équilibre dynamique d'un élément de chacune des barres permet d'exprimer à tout instant le déplacement et la charge moyenne dans l'échantillon en fonction des différentes ondes (incidente, réfléchie et transmise) par [6]

$$
\begin{aligned}
& d s(t)=c_{0} \int_{0}^{t}-\left[\varepsilon_{1}(t)+\varepsilon_{R}(t)+\varepsilon_{T}(t)\right] d t \\
& P_{S}(t)=A_{S} \quad\left[\varepsilon_{1}(t)+\varepsilon_{R}(t)+\varepsilon_{T}(t)\right]
\end{aligned}
$$

avec Co la célérité des ondes mécaniques dans le milieu considéré ef $A_{S}$ la section des barres.

Du fait du caractère amortissant de ce genre de matériau, lhypothèse quasi-statique qui suppose qu'à tout instant les efforts sur chaque extrémité de l'échantilion sont égaux peut-être discutée. En ce sens un phénomène de désadaption d'impédance mécanique ( $\rho C A)$ avait déjà été observé par NAIT [ 6 ] sur un matériau comparable. C'est pourquoi une faible hauteur d'échantillon $(7 \mathrm{~mm})$ a été choisie au détriment d'une certaine triaxialité des contraintes en fond de fissure.

Finalement ce dispositif permet l'obtention de vitesse de déformation jusqu'à environ $300 \mathrm{~s}^{-1}$ (à comparer à $10^{3} \mathrm{~s}^{-1}$ pour les métaux du fait de l'amortissement).

\section{THEORIE GENERALISEE DE MECANIQUE DE LA RUPTURE}

Cette théorie propose une méthode de détermination du paramètre énergétique -dU/dA où U est l'énergie potentielle du système considéré et A la surface de la fissure qu'il contient.

Brièvement, en supposant un problème plan et un système de dimensions infinies contenant un défaut de longueur a (surface $A=2 B a, B$ largeur), la densité locale d'énergie de déformation s'écrit en un point $P$ quelconque en fonction de la densité uniforme loin du défaut, Wo: 


$$
W(P)=W_{0} f\left(x / a, y / a, \varepsilon_{0}\right)
$$

A remarquer l'utilisation des coordonnées réduites $x / a, y / a$ de façon à résoudre le problème dans un espace sans dimension. Bien évidemment si l'échantilion n'est plus de dimensions infinies, il faut faire intervenir dés cette étape des termes correctifs dépendant de la géométrie.

La variation de cette densité consécutive à la propagation du défaut sur une longueur da (a Wo constant) s'écrit alors :

$$
\mathrm{dW}(\mathrm{P}) / \mathrm{da}=-\mathrm{Wo} / \mathrm{a} \quad \mathrm{g}\left(\mathrm{x}, \mathrm{y}, \varepsilon_{\mathrm{o}}\right)
$$

où $g$ est une nouvelle fonction faisant intērvenir les dérivées partielles de la fonction f. La sommation sur l'ensemble des points $P$ permet d'accéder à la variation totale d'énergie dans le système et en supposant un comportement réversible cette énergie est caractéristique de la rupture :

$$
d U / d a=\sum_{p} d W(P) / d a \quad \delta v=-B W o \text { a } \sum_{p} g\left(x, y, E_{0}\right) \delta x \delta y
$$

$\left(\delta v=a^{2}\right.$ B $\delta x \quad \delta y$ pour le probleme plan). La sommation s'effectuant dans un espace sans dimension et à Wo, $\mathcal{E}_{0}$ constant, on peut poser :

$$
\sum_{p} g\left(x, y, \varepsilon_{0}\right)=k_{1} \quad\left(W_{0}\right)
$$

Cette fonction caractérisant alors la distribution de la densité d'énergie de déformation et donc les effets de non-linéarité. C'est d'après ANDREWS une caractéristique intrinsèque du matériau.

Le critère énonce donc:

$$
J=-d U / d A=k_{1}\left(W o_{c}\right) \cdot W o_{c} \cdot a \cdot
$$

où Wo $\mathrm{W}_{\mathrm{c}}$ est la valeur critique de Wo. Dans notre cas, la géométrie est axisymétrique et la relation devient :

$$
J=-d U / d A=k_{1}(W o) . \text { Wo. } a^{2} / 2 \pi(R-a)
$$

où $\mathrm{R}$ est le rayon de l'éprouvette considérée.

Expérimentalement on mesure des quantités $\Delta U, a$, wo constant sur les courbes charge-déplacement obtenues pour différentes longueurs de défaut et la fonction $k_{1}$ (Wo) est obtenue par intégration de la relation (7) dans laquelle on fait intervenir une correction de géométrie du même type que les fonctions de calibration utilisées pour le calcul du facteur d'intensité de contrainte $K$. 
Finalement la ténacité est déterminée pour les conditions critiques (dans notre cas la charge maximale) en plaçant les points $k_{1}\left(W_{O_{C}}\right)$. Wo ${ }_{c}$ en fonction de $\left[a^{2} / 2 \pi \text { (R-a) }\right]^{-1}$ qui s'alignent sur une droite de pente Jc.

\section{RESULTATS - DISCUSSION}

La figure 4 montre les différentes étapes de la méthode.

On observe une grande dispersion dans la fonction $k_{1}$ (Wo) qui ne semble pas une caractéristique intrinsèque du matériau mais conserve une dépendance vis-à-vis de la longueur du défaut. A n'en pas douter, la correction de géométrie que nous avons utilisée est inadaptée au comportement non-linéaire du matériau. Cependant il n'existe pas dans la littérature de fonction de calibration non-linéaire ef il sera nécessaire d'effectuer un calcul aux éléments finis pour conclure définitivement sur ce point.

Toutefois quelques commentaires qualitatifs sont à faire particulièrement dans le domaine des faibles Wo. En effet, théoriquement, sous de très faibles charges tous les matériaux ont un comportment linéaire et dans ce cas:

$$
-d U / d A=G=\pi . \text { Wo. } a
$$

La fonction $\mathrm{k}_{1}$ (Wo)devrait donc tendre asymptotiquement vers la valeur $\pi$ pour Wo $\rightarrow 0$.

Ce qui laisse supposer que pour ce matériau, $k_{1}$ augmente très rapidement pour atteindre une valeur qui n'est plus que légèrement croissante dès l'apparition de la décohésion. Malheureusement les mesures expérimentales sont assez délicates sur un tel matériau dans ce domaine (problèmes de flexion et d'hétérogéneïté).

Malgré tout on observe un assez bon alignement des points expérimentaux sur la droite ce qui conduit aux résultats du tableau $\mathrm{B}$ qui récapitule les valeurs de $\mathrm{J}$ pour les différentes granulométries.

$\begin{array}{lrrrr}\text { Granulométrie } & \text { Go } & \text { G1 } & \text { G2 } & \text { E0 } \\ \text { Ténacité }\left(\mathrm{kJ} / \mathrm{m}^{2}\right) & 3,69 & 5,72 & 1,89 & 2,05\end{array}$

Tableau B

On observe une sensibilité importante de la ténacité de ce matériau à la répartition granulométrique des charges qu'il contient. De plus la granulométrie la plus fine conduit à un maximum de ténacité, ce qui indique que la forte hétérogénéité est nuisible à sa tenue mécanique. A remarquer que les granulométries repérées E et G2 conduisent sensiblement à la même ténacité, la répartition des charges de 30 et $8 \mu$ semble donc n'avoir que peu d'influence sur la ténacité. La rupture s'amorce donc préférentiellement aux gros grains et la ténacité sera 
d'autant meilleure que la dimension de ces plus grosses charges sera faible. (Cependant d'un point de vue énergétique ces granulométries possèdent de meilleures caractéristiques de combustion et il serait donc nécessaire d'établir un compromis résistance mécanique/pouvoir calorifique).

\section{CONCLUSIONS}

La ténacité de carburants solides de diverses granulométries a été testée sous sollicitation dynamique et la sensibilité du matériau à ce paramètre a été mise en évidence. Les résultats expérimentaux permettent d'envisager un processus de rupture essentiellement intergranulaire s'amorçant de façon préférentielle sur les gros grains. Inversement les caractéristiques énergétiques optimales sont obtenues pour ces gros grains. Cependant une autre étude a mis en évidence que les sollicitations dynamiques conduisent à des ruptures partiellement transgranulaires [ 7 ]. La suite de cette étude expérimentale concernera donc la rupture du polymère utilisé comme liant de façon à mieux cerné la participation de celui-ci au processus de rupture.

Nous remercions Mr Arnaudas et la Société Brandt pour l'aide apportée à ce travail.

\section{REFERENCES}

[1] RIVLIN R.S, THOMAS A.G, Rupture of Rubber, Journal of polymer Science Vol $10, N^{\circ} 3$, 291-318 (1953).

[2] E.H ANDREWS, A generalized theory of fracture mechanics, Jour. of mat. Sci., 9, 887-894 (1974).

[ 3 ] Recommandation DYMAT. Méthodes d'essais : utilisation des barres d'Hopkinson (à paraître)

[4] KLEPACZKO J.R, Application of the split Hopkinson pressure bars to fracture dynamics, inst. Phys. Conf. Sci. № 47, Chap 2, p. 201, Ed. J. HARDING, Oxford (1979).

[5] H. KOLSKY, An investigation of mechanical properties at high rate of loading Proc. Phys. Soc. 62, 676-700, (1949).

[6] M. NAIT-ABDELAZIZ, R.G NEVIERE, Experimental method for $J_{/ c}$ computation on fracture of solid propellants. Eng. Fract. Mech. Vol 28, $N^{\circ} 4,425-434$, (1987).

[7] Ph BOULE, Contribution de la fractographie quantitative à l'étude de la rupture dynamique d'un propergol. Thèse de doctorat présentée à l'Université de METZ le 04 Mars 1988. 


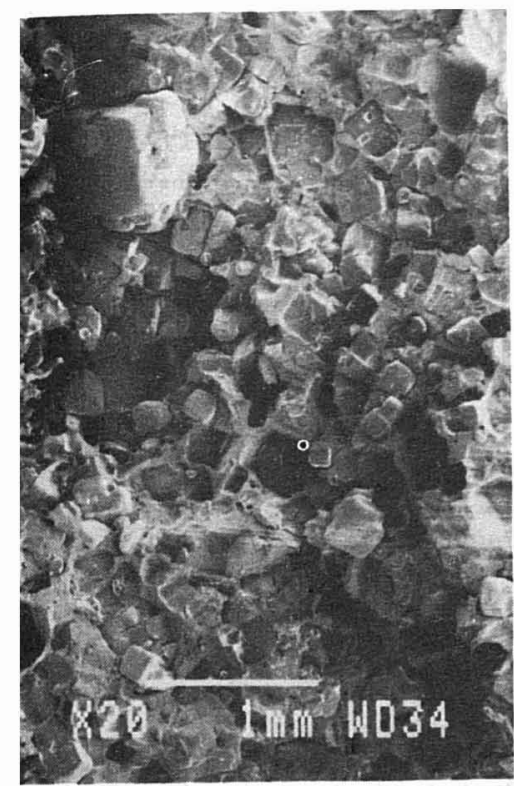

Fig. i : Structure d'un propergol

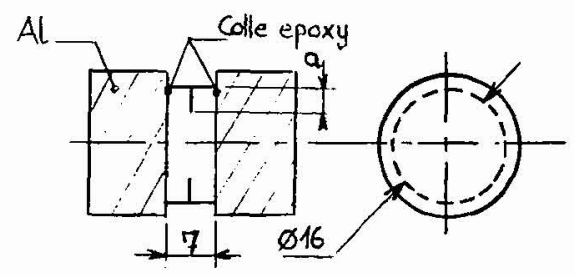

Fig. 2 : Echantillon

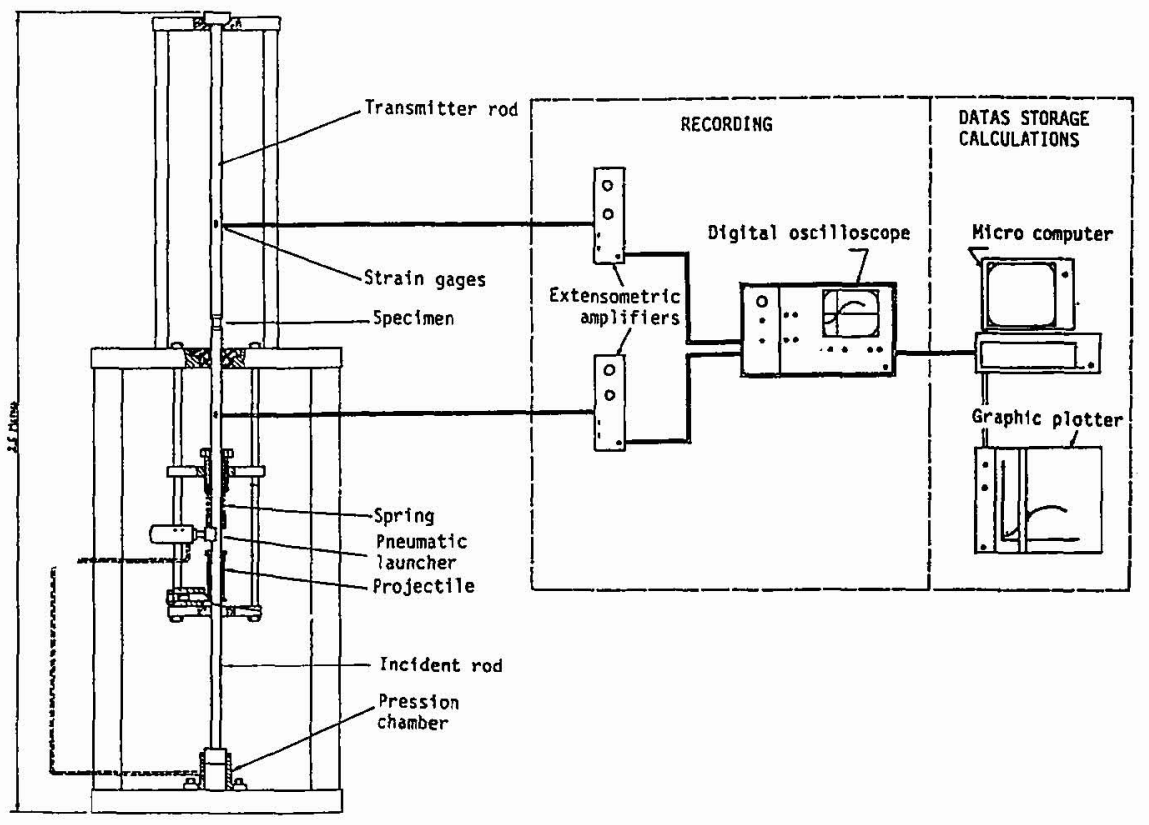

Fig. 3 : Dispositif de traction dynamique 

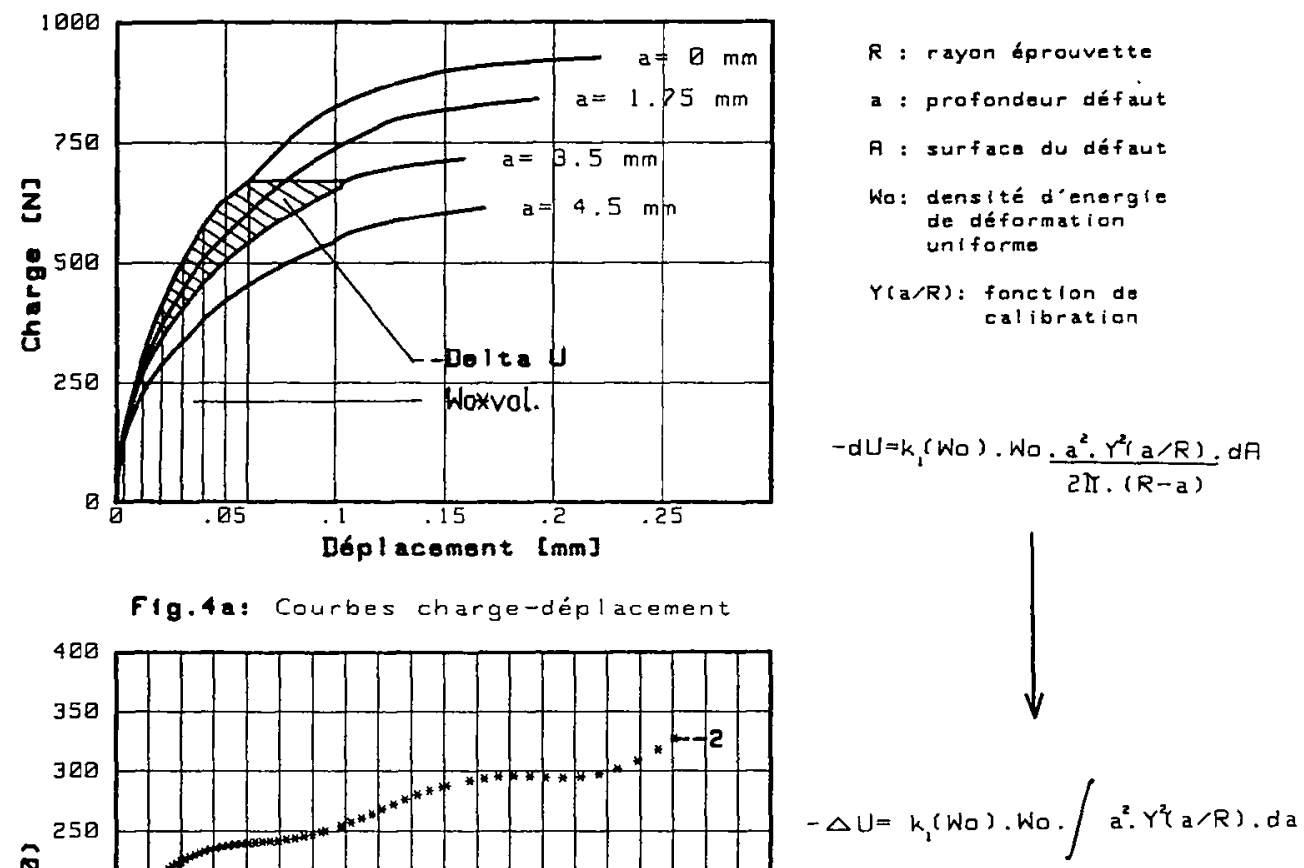

$-\Delta U=k_{1}\left(W_{0}\right) \cdot W_{0} \cdot \int a^{2} \cdot Y^{2}(a / R) \cdot d a$

Fig.4a: Courbes charge-déplacement

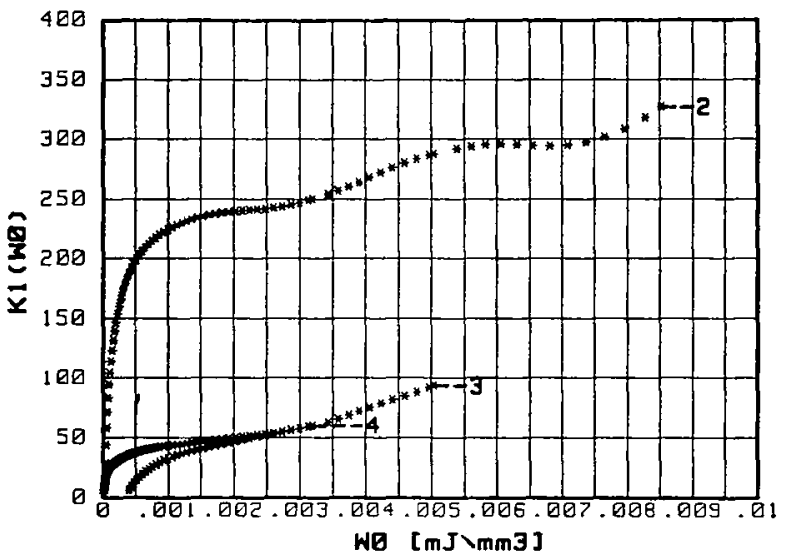

Fig.4b: Fonction KI(WO)

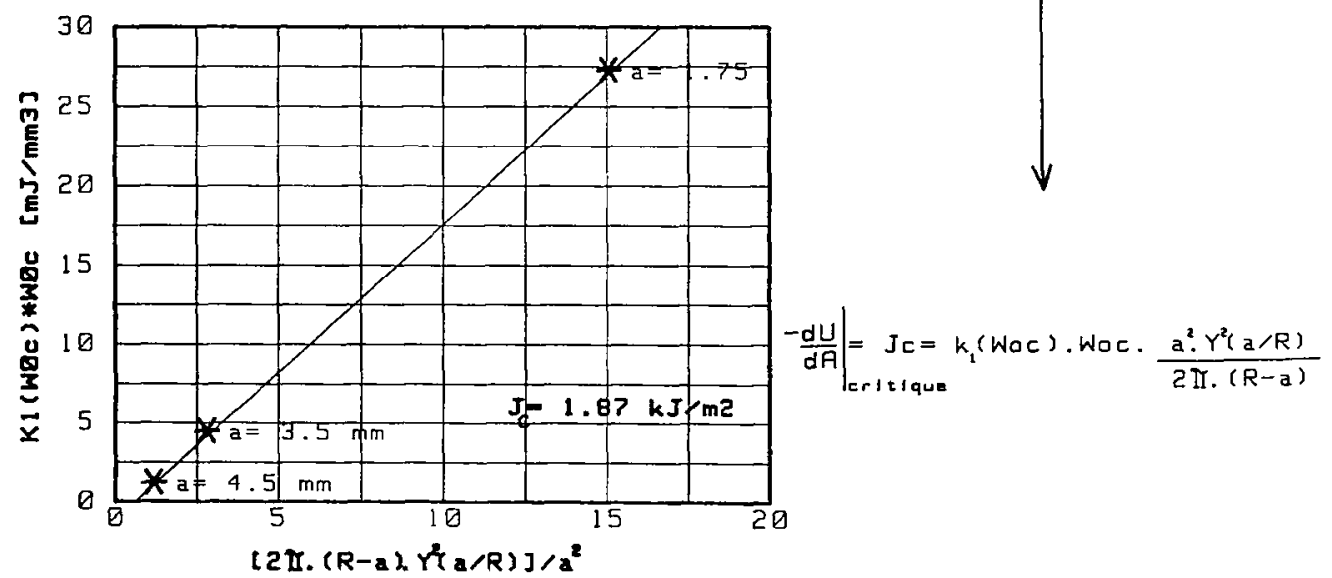

Fig.4c: Droite d'ANDREWS 